\title{
Fracturation naturelle d'un massif rocheux Diaclases et couloirs de fracturation
}

\author{
C. Putot ${ }^{1}$, J. Chastanet ${ }^{1 *}$, M.C. Cacas ${ }^{2}$ et J.M. Daniel ${ }^{2}$ \\ 1 Division Mécanique appliquée, Institut français du pétrole, 1 et 4, avenue de Bois-Préau, 92852 Rueil-Malmaison Cedex - France \\ 2 Division Géologie-Géochimie, Institut français du pétrole, 1 et 4, avenue de Bois-Préau, 92852 Rueil-Malmaison Cedex - France \\ e-mail : claude.putot@ifp.fr \\ * Actuellement Laboratoire 3S Sols-Solides-Structures, Domaine universitaire, BP 53, 38041 Grenoble Cedex 9 - France
}

Résumé - La caractérisation de la fracturation des réservoirs passe par l'estimation d'écartements typiques entre fractures constituées en mode d'ouverture. L'échelle dépend de la configuration de chargement et des propriétés mécaniques supposées de la roche : résistance à la rupture, énergie de décohésion, etc. La présente étude porte sur une morphologie de fractures particulière : les couloirs de fracturation. Ce sont des zones de forte densité de fractures apparaissant régulièrement dans les bancs épais et compacts. L'étude sur les couloirs de fracturation se situe dans le prolongement d'une analyse récente concernant les diaclases dont on a recherché l'espacement moyen. On a défini dans les deux cas, pour la roche, les paramètres mécaniques pertinents permettant de fixer l'échelle des distances entre fractures.

Dans le cas des diaclases, la fracturation se présente de manière assez homogène, l'échelle étant dictée en grande partie par l'épaisseur des bancs, quoique influencée de manière secondaire par la nature de la roche et le chargement au loin.

Dans le cas de la fracturation interne à un couloir, les rôles respectifs d'une surface libre préexistante et de concentrateurs de contraintes sont invoqués, le rôle des bancs devenant accessoire : en effet, cette typologie peut même exister dans les roches ignées. Une diaclase majeure, présentant une certaine persistance vis-à-vis des bancs, peut jouer le rôle de surface libre. Dans ces conditions, la morphologie des fractures montre dès lors une allure plus hétérogène. Cette condition aux limites particulière induit, transversalement à la surface libre, un « gradient d'états » se traduisant par une certaine distribution de longueurs de fractures et d'écartements.

L'échelle caractéristique des fractures et le rapport de forme/longueur sur écartement ont été calculés en fonction du confinement par un modèle explicatif simple. On tend, à la surface libre, vers des fractures continues et d'écartement donné. De manière duale, en marge du couloir, on note une limite du facteur de forme, limite déterminée par les caractéristiques de la roche.

Afin de déterminer les caractéristiques de ces structures (élancements, espacements, etc.), nous avons fait appel à des modèles de fractures dont la géométrie restitue localement celle des couloirs. De plus, un facteur d'échelle a été détecté.

Les modèles se rangent en deux catégories : ceux qui, outre le paramètre de ténacité de la roche, mettent en lumière le rôle du module élastique de la roche, et ceux faisant intervenir une limite à la rupture. Les premiers donnent des élancements élevés (fractures rapprochées) tandis que les seconds nous semblent beaucoup plus pertinents dans la description des rapports de forme (plus massifs).

Ces modèles ont permis de conjecturer l'image des couloirs et de discuter le réalisme, du point de vue géologique, du mécanisme de formation établi ici.

Mots-clés : mécanique de la rupture, couloirs de fracturation, diaclases, hétérogénéité de contraintes, flambage, plasticité, confinement, surface libre, facteur d'échelle, rapport de forme. 


\begin{abstract}
Fractography in Sedimentary Rocks: Tension Joints Sets and Fracture Swarms - In order to characterize fracturation in reservoirs, one must estimate typical opening-mode fracture spacing, whose magnitude depends on the loading configuration and the mechanical parameters for the rock, primarily strength and cohesion energy. This study focuses on a specific fracture typology known as fracture swarms, which consist of heavily fractured rocks that regularly appear in thick compact layers.

This analysis of fracture swarms is the continuation of a recent study of tension joint sets. In both cases we have defined the mechanical parameters necessary to determine typical fracture spacing.

In the case of regular joint spacing, the fracturation is rather homogeneous, and the typical length scale depends primarily on the thickness of the layers, although it also depends on the type of rock and on remote loading.

In the case of fracture swarms, the roles of both a preexisting free surface and of some form of stress concentrator are discussed. In this case, the bed thickness plays a subordinate part, and, indeed, this kind of fractography can exist in igneous rocks. A major joint that has propagated across beds could act as a free surface. When this is the case, the morphology of fractures is more heterogeneous. This kind of boundary condition generates, perpendicular to the free surface, a state gradient that expresses itself as a distribution of fracture lengths and spacings.
\end{abstract}

Characteristic fracture scaling and aspect ratios (length/spacing) have been calculated as a function of confinement using a simple generic model. The asymptotic behavior near the free surface is a prescribed spacing and indeterminate fracture length. However, at the limit of the outcrop, a clearly defined aspect ratio has been found with an indeterminate spacing. These limits are determined as well defined functions of the mechanical properties of rock.

The determination of aspect ratios and spacing has been carried out with the help of fracture models that mimic what one expects to see generated at a local scale. Moreover, scaling factors have been inferred.

Models have been classified in two categories, those that, in addition to the toughness parameter, consider the elastic modulus of rock as the pertinent parameter and those for which the key is, rather, some measure of strength. The former, by some kind of predominant buckling mechanism, lead to underestimated spacing values, whereas the latter seem to better express both aspect ratios and the order of magnitude of the spacing.

These models have allowed us to conjecture a picture of the distribution of fracture swarms and to discuss their significance in the formation process, from the geologist's standpoint.

Keywords: fracture mechanics, fracture swarms, joint sets, stress concentration, buckling, plasticity, triaxiality, free surface, scale effects, aspect ratio.

\section{INTRODUCTION}

La fracturation naturelle des réservoirs d'hydrocarbures regroupe toutes les discontinuités d'origine mécanique affectant la matrice rocheuse. Ces fractures naturelles constituent le plus souvent des drains préférentiels ou des barrières de perméabilité qui contrôlent en partie le déplacement des fluides en cours de production. En cela, l'étude de la fracturation constitue un élément de tout premier ordre en caractérisation des réservoirs.

Les roches se fracturent naturellement selon une zone de déformation en cisaillement (les plans de rupture correspondants sont les failles) ou en rupture fragile (les plans de rupture correspondants sont les diaclases). Les deux types de fracturation ont fait l'objet d'observations géologiques depuis plusieurs décennies et ont permis d'accumuler de nombreuses connaissances empiriques et qualitatives (Pollard et Aydin, 1988). Des approches mécaniques et quantitatives ont également été proposées (Atkinson, 1987), mais demandent à être complétées, notamment en ce qui concerne le développement des réseaux de diaclases. C'est ce dernier point qui fait l'objet du présent article.

\section{Diaclases systématiques et couloirs de fracturation}

Les diaclases (fig. 1) apparaissent à l'affleurement comme des traces généralement continues et rectilignes, dont les lèvres, apparemment jointives, ne présentent aucune trace de mouvement cisaillant des deux compartiments. Les réseaux de diaclases peuvent présenter des morphologies très différentes d'un site à un autre. En particulier, ils peuvent se présenter en réseaux très réguliers en termes d'espacement et d'orientation (dits réseaux de diaclases systématiques), ou au contraire avec une répartition spatiale beaucoup plus hétérogène, présentant des couloirs de fractures (fig. 2). Ces derniers se présentent comme des regroupements, sur une zone assez bien délimitée, de diaclases à fort développement 


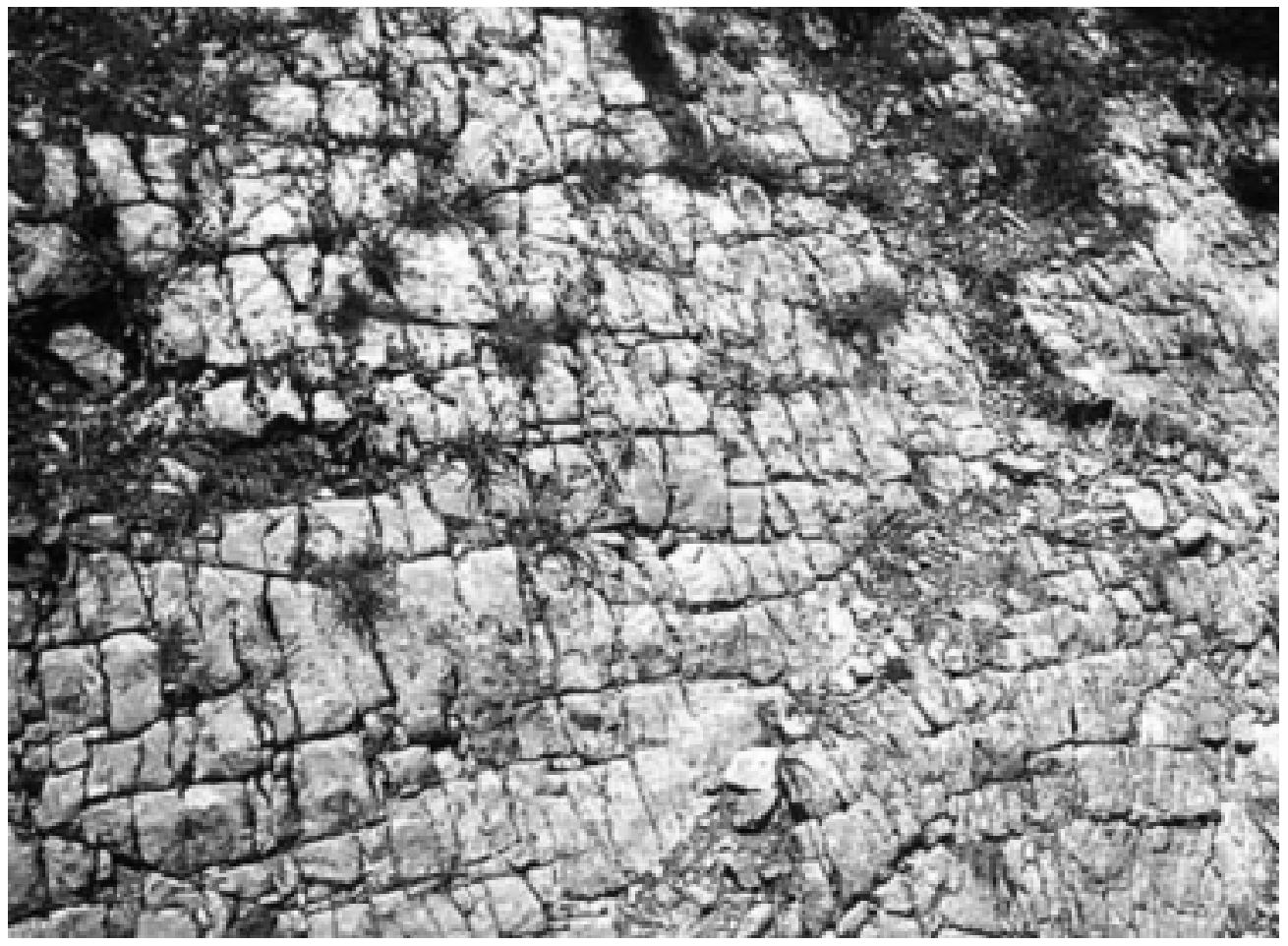

Figure 1

Réseau de diaclases sur les dalles de marno-calcaires du Berriasien inférieur du flanc ouest de l'anticlinal du couvent, au Col de la Ruchère. Joint set on the structural surface of shaly limestone lower Berriasian on the west flank of the convent anticline, near the Col de la Ruchère.

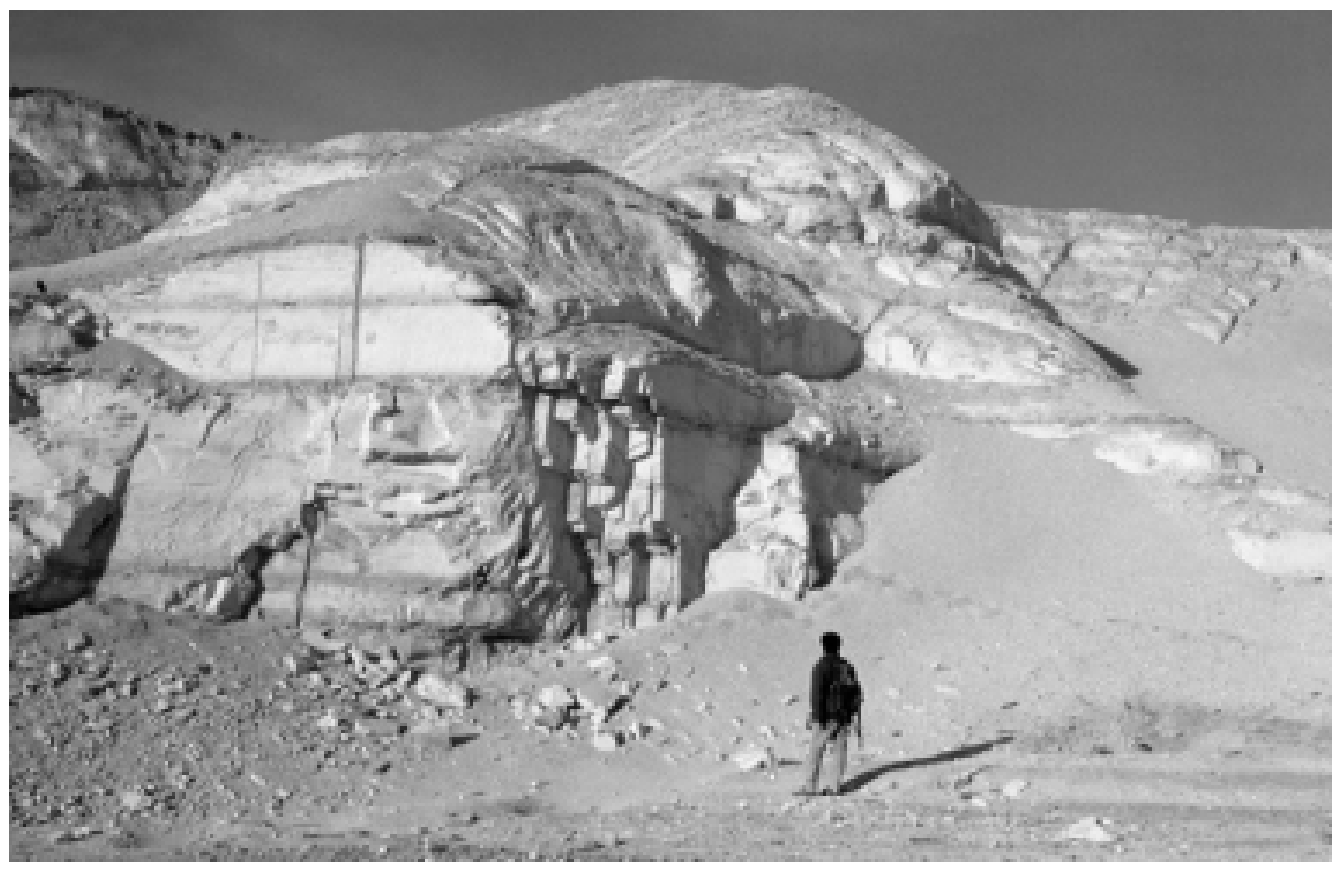

Figure 2

Photographie d'un couloir.

A fracture swarm. 
vertical, dit persistance. Les couloirs se différencient des réseaux réguliers, d'une part, par le fait que la fracturation y est localisée et d'autre part, par leur dimension caractéristique : les fractures des couloirs traversent plusieurs bancs tandis que le développement vertical des diaclases systématiques semble fortement contrôlé par ces limites de bancs.

Jusqu'à ce jour, les couloirs de fracturation ont rarement été décrits et expliqués dans la littérature. Plusieurs définitions ont cependant été proposées :

- Définition de Rives (1992) : il s'agit d'un amas de diaclases d'une même famille qui se relaient pour donner une continuité verticale apparente à travers plusieurs bancs.

- Définition de Auzias (1995) : on parle de couloir fracturé lorsque quelques diaclases majeures sont suffisamment rapprochées pour former une zone aux limites bien précises qui est souvent déprimée par l'érosion. Ces diaclases majeures, assez régulièrement espacées et parallèles, sont peu connectées entre elles.

Les couloirs découpent en général des affleurements dont l'extension horizontale peut être de plusieurs kilomètres et dont la hauteur peut atteindre plusieurs centaines de mètres. Ils sont observés dans les roches sédimentaires et parfois dans le granite, mais toujours dans des séries compactes. Le litage peut jouer un rôle important dans la localisation des couloirs : dans les roches litées, la localisation est moindre, les couloirs sont plus larges. Les couloirs peuvent être associés à une faille ou à un dyke.

En résumé, un couloir est une famille de diaclases regroupées sur une zone étroite. Les observations ne permettent pas d'en donner une taille caractéristique: pas d'écartement typique, longueurs de fractures variables. Leur trait commun est le fort degré de continuité (persistance verticale) malgré les litages : les fractures se sont propagées à travers plusieurs bancs.

Il est important de savoir quantifier et de mieux connaître les facteurs contrôlant ces deux répartitions spatiales très différentes. Si les diaclases systématiques permettent de produire des réservoirs compacts, inexploitables sans fissuration, les couloirs de fractures, au contraire, sont responsables d'arrivées d'eau précoces très préjudiciables. La stratégie d'implantation des puits sera différente suivant le type de fracturation en présence. Cependant, le géologue dispose de très peu d'indices permettant de déterminer directement le type de fracturation, systématique ou en couloirs, qui affecte un réservoir donné. L'étude décrite ci-dessous tente d'apporter des éléments de réponse théoriques et quantitatifs.

La présente analyse vise à caractériser les couloirs de fracturation par rapport aux systèmes de diaclases.

\section{PROPOS ET ORGANISATION DE LA PRÉSENTE ÉTUDE}

Les mécanismes conduisant à la formation des couloirs de zones fracturées paraissent analogues à ceux qui expliquent la propagation des diaclases majeures, mais un facteur complémentaire doit cependant être invoqué pour justifier une densité de fracturation a priori différente.

Auzias (1995) a proposé trois explications faisant toutes allusion à des considérations d'hétérogénéité de répartition des contraintes, autrement dit, de concentration locale des efforts :

- l'augmentation locale de la pression fluide peut provoquer la formation de plusieurs diaclases sur une petite zone formant ainsi un couloir ;

- le paléorelief du socle, de par son hétérogénéité, peut initier des couloirs dans la couverture sédimentaire ;

- la relaxation des contraintes le long des bords libres d'une diaclase majeure préexistante peut favoriser la formation d'autres diaclases à proximité.

Nous nous proposons de montrer comment l'usage de modèles mécaniques explicatifs de géométrie élémentaire permet, moyennant certaines hypothèses vraisemblables, de prédire des physionomies de rupture compatibles avec les observations, notamment l'écartement et le degré de persistance (continuité au-delà des litages). Ces modèles mettent en jeu des paramètres liés aux propriétés de la roche et à l'échelle des bancs lorsqu'il s'agit d'horizons sédimentaires.

Sur le plan de la mise en œuvre pratique, deux procédés de calcul assez différents ont été utilisés. Le premier, suivant la stratégie d'analyse de Kemeny et Cook (1985), est appliqué aux diaclases ; le second concerne le couloir de fracturation.

- Le premier procédé, utilisant le cadre de la mécanique linéaire de la rupture, prend en compte un schéma de banc rocheux susceptible de contenir un grand nombre de fractures parallèles, perpendiculaires aux litages, non débouchantes mais saisies dans leur état propagé, à l'arrêt (on ne s'interroge pas sur la phase d'amorçage). Supposant la fracturation périodique et les fractures toutes identiques, l'inconnue est principalement la densité de fractures et, accessoirement, la longueur de la fissure.

- La seconde méthode utilise les résultats généraux (Puttick, 1979) obtenus en matière de transition de mécanismes ductile/fragile (Atkins et Mai, 1985). La taille recherchée pour le motif délimitant deux fractures contiguës est celle pour laquelle le mécanisme de ruine ne peut plus être la plasticité. (On sait que des éprouvettes homothétiques de grandes dimensions privilégient la rupture fragile, la rupture nécessitant alors la fourniture d'une énergie de surface plus modérée que la plasticité dont les besoins ne sont satisfaits que sur un volume).

On émet l'hypothèse, dans toute cette présentation, que roches et minéraux se prêtent au cadre de la mécanique élastique linéaire de la rupture (Lachenbruch, 1961). La caractéristique contrainte/déformation de certaines roches est considérée comme non linéaire (Scholz, 1990) et l'on peut alors s'interroger sur la validité d'une telle approximation. On a pu constater néanmoins, pour des températures qui ne sont pas 
trop élevées, que les paramètres linéaires étaient compatibles avec les valeurs de l'intégrale $J$ qui ne présuppose pas un cadre si restrictif (Atkinson, 1984).

En fait, le géologue préfère à la définition usuelle du facteur d'intensité de contraintes critique $K_{I C}$ impliquant une propagation catastrophique, une vue adaptée au chargement à long terme, qui malgré tout peut être de courte durée vis-à-vis des temps géologiques (Bahat, 1991). Le phénomène visé est la croissance sous-critique de fissures appelée encore propagation quasi statique ou stable. Ce phénomène résulte de plusieurs mécanismes en compétition, dont certains sont sous la dépendance de la chimie de l'environnement, le facteur le plus connu étant la corrosion sous contrainte. La croissance sous-critique est caractérisée par des vitesses qui décroissent très sensiblement avec le facteur d'intensité de contraintes $K_{I}$. Le seuil de propagation correspond à une valeur $K_{0}$ de l'ordre de 10 à $20 \%$ de $K_{I C}$.

On pourra se reporter à l'annexe 2 pour un aperçu des facteurs conditionnant le paramètre de ténacité des roches, notamment la dépendance vis-à-vis de la taille de grain. L'annexe 3 établit le lien entre ce paramètre mécanique et d'autres, plus familiers des mécaniciens des roches. L'annexe 4 donne quelques indications sur les dispositifs de mesure de ce paramètre. Certains sont très élaborés et précis mais nécessitent la mise en œuvre de moyens importants. D'autres sont sommaires, mais peuvent être utilisés sur le terrain.

Dans ce qui suit, on ignore le facteur temporel qui préside à la progression lente d'un fissure en situation de chargement $K_{I}<K_{I C}$ et on se place en situation de propagation brutale $K_{I}$ $=K_{I C}$. En fait, la physionomie supposée de l'évolution du facteur d'intensité des contraintes en fonction de la profondeur atteinte par le front de fissuration est telle qu'il y a arrêt de la fissure.

\section{RÉSEAUX DE DIACLASES NON LOCALISÉS}

Les diaclases (Pascal et al., 1997) sont étudiées en milieu homogène ; l'unité structurale est un banc d'épaisseur $H$ et d'extension horizontale quelconque mais suffisante pour ne pas créer de problèmes de bord dans la modélisation. On a donc considéré, avec Kemeny et Cook (1985), un modèle de couche homogène en déformations contrôlées (voir par exemple Francfort et Marigo, 1998, pour la justification d'un chargement en déformations contrôlées) à deux paramètres de chargement. L'un prend en compte l'effet de la pression hydrostatique et soumet la poutre à une contraction isotrope $\varepsilon$, l'autre est une courbure imposée uniforme sur la longueur de la poutre (fig. 3). Un paramètre de chargement noté $\lambda$ caractérise le mécanisme tectonique supposé ; il exprime, au sein du réservoir, le degré de confinement comparé à l'effet de flexion.

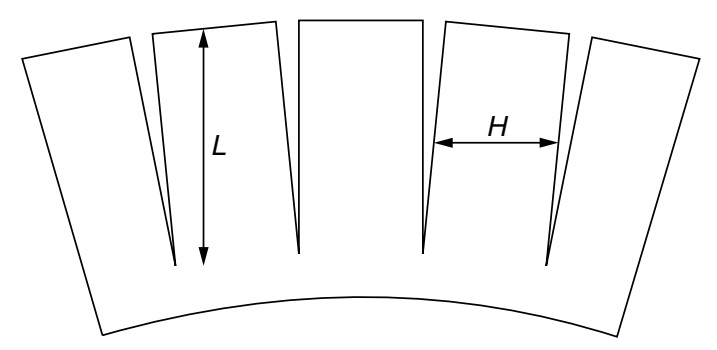

Figure 3

Fracturation régulière (longueur $L$, écartement $H$ ) dans un massif chargé en contraction isotrope et en courbure.

Regular cracking (length L, spacing $H$ ) in a bedded geologic setting loaded with a constant curvature and hydrostatic deformation.

\subsection{Méthode de calcul de la densité de fissuration}

La méthode de calcul consiste à déterminer le facteur d'intensité de contraintes $K_{I}$ associé à la condition de chargement ou (sa valeur réduite $\kappa=K_{I} / \sigma_{0} \sqrt{ } H$ où $\sigma_{0}$ est la limite de rupture en traction de la roche) en fonction de la longueur relative de fissure $L / H$, à chargement $\lambda$ donné, et ceci pour diverses valeurs de la densité de fissuration supposée (les effets d'interaction entre fissures voisines sont cependant négligés quelle que soit la densité sélectionnée). Densité et profondeur de fissuration définissent une répartition entre énergie élastique associée à la déformation de la matrice rocheuse et énergie associée à l'ouverture de chacune des fissures. La configuration choisie par le processus de fracturation - parmi toutes celles calculées - sera celle exigeant, pour sa réalisation, un minimum d'énergie, d'où l'équidistance recherchée et la profondeur d'arrêt de la fissuration associée.

Par exemple, la déformation de la matrice rocheuse est nécessairement importante si les fissures sont peu nombreuses, ce qui consomme peu d'énergie si la roche est plastique «ductile». Au contraire, si la ténacité est faible (roche fragile) une forte densité de fissuration sera privilégiée par le procédé d'optimisation, car la fracturation sera peu consommatrice d'énergie de surface.

De ce fait, le calcul comporte deux étapes :

- Supposant une certaine densité de fracturation, on détermine dans un premier temps la profondeur atteinte $L$ : l'arrêt/stabilisation est justifié par la décroissance de la courbe $\kappa(L / H)$ au-delà du maximum (fig. 4) ; $L$ est calculée comme abscisse de l'intersection de cette portion de courbe avec l'horizontale associée à la ténacité réduite.

- Cette seconde étape aboutit à la densité de fissuration recherchée par simple minimisation de l'énergie associée au passage de la configuration vierge à la configuration finale fissurée : on notera que le critère de Griffith considéré 


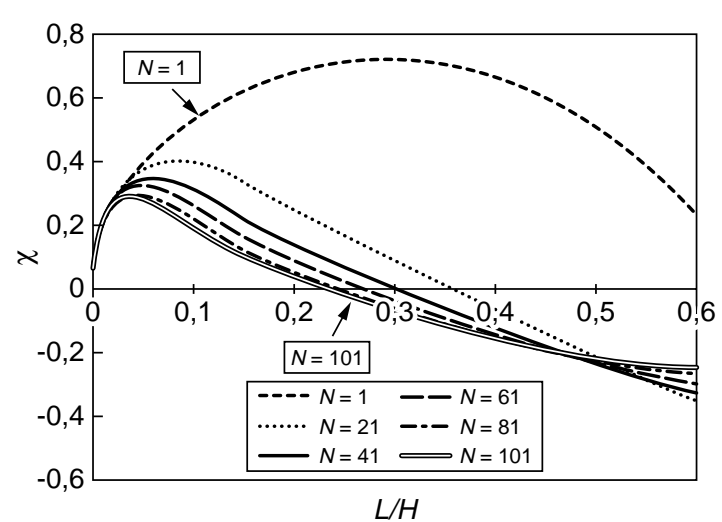

Figure 4

Évolution de $\kappa=\frac{K_{I}}{\sigma_{0} \sqrt{H}}$ en fonction de la profondeur relative de diaclase $L / H$ - courbes paramétrées par $N$, densité de fissures (d'après Fournigault, 2000).

Variation of $\kappa=\frac{K_{I}}{\sigma_{0} \sqrt{H}}$ as a function of relative length

$L / H$, with curves characterized by $N$, the crack density (after Fournigault, 2000).

habituellement concerne un accroissement infinitésimal de la fissuration, tandis que le critère proposé ici - en transformation finie - en est une extension (Francfort et Marigo, 1993).

\subsection{Résultats}

L'épaisseur des bancs $H$ induit évidemment une échelle naturelle qui conditionne directement la valeur des écartements $h$ recherchés.

Le deuxième facteur déterminant concerne la nature de la roche, saisie par sa ténacité réduite :

$$
\kappa_{C}=\frac{K_{I C}}{\sigma_{0} \sqrt{H}}
$$

(On omettra l'indice $C$ dans ce qui suit). En fait, il est plus pratique de constater que $(\kappa \sqrt{H})^{2}$ est proportionnel à une taille de zone plastique (endommagée) notée $d^{*}$. On écrit ainsi :

$$
\kappa^{2} H \propto d^{*} \propto\left(\frac{K_{I C}}{\sigma_{0}}\right)^{2}
$$

Nos résultats (Fournigault, 2000) indiquent pour les espacements $h$ et les profondeurs atteintes $L$ les dépendances suivantes vis-à-vis de l'épaisseur $H$ :

$$
\frac{L}{H} \propto \kappa \text { soit }: \frac{L}{H} \propto\left(\frac{d^{*}}{H}\right)^{1 / 2} \text { soit }: L \propto\left(d^{*} H\right)^{1 / 2}
$$

$$
\begin{aligned}
& \frac{h}{H} \propto \kappa^{3} \text { soit }: \frac{h}{H} \propto\left(\frac{d^{*}}{H}\right)^{3 / 2} \text { soit }: h \propto\left(\frac{d^{* 3}}{H}\right)^{1 / 2} \\
& \frac{h}{L} \propto \kappa^{2} \text { soit }: \frac{h}{L} \propto\left(\frac{d^{*}}{H}\right) \text { soit }: \frac{h}{L} \propto\left(\frac{d^{*}}{H}\right)
\end{aligned}
$$

L'étape suivante - qui permet de conclure de manière concrète - est associée à l'écriture d'une hypothèse d'une variation supposée de $\kappa$, paramètre mécanique, ou, de manière équivalente $d^{*}$, avec $H$.

\subsubsection{Hypothèse 1}

Supposant $K_{I C}$ invariable avec l'échelle des bancs, on admet pour cette première hypothèse que :

$$
\sigma_{0} \propto H^{-1 / 2} \text { soit }: d^{*} \propto H
$$

Dans ces conditions, on obtient les résultats suivants :

$$
\begin{gathered}
L \propto H \propto d^{*} \\
h \propto H \propto d^{*} \\
\frac{L}{h} \propto 1
\end{gathered}
$$

Le deuxième résultat - proportionnalité des écartements moyens $h$ à l'épaisseur du banc $H$ - est fréquemment observé (fig. 5) lorsque $H$ demeure modéré (Ladeira et Price, 1981).

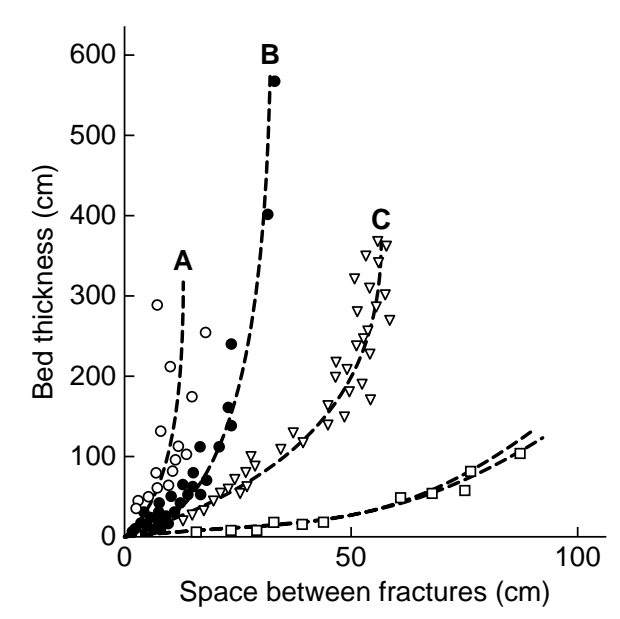

Figure 5

Base de données de Ladeira et Price (1981) montrant la saturation des écartements vers les bancs de forte épaisseur (voir hypothèses 1 et 2 ci-après).

Data according to Ladeira and Price (1981) exhibiting saturation of spacing with increasing thickness (see Hypotheses 1 and 2 below). 


\subsubsection{Hypothèse 2}

Supposant également $K_{I C}$ invariable avec l'échelle des bancs, on admet désormais que :

$$
\sigma_{0} \propto H^{-1 / 6} \text { soit }: d^{*} \propto H^{-1 / 3}
$$

Dans ces conditions :

$$
\begin{gathered}
L \propto H^{2 / 3} \propto d * 2 \\
h \propto 1 \\
\frac{L}{h} \propto H^{2 / 3} \propto d^{* 2}
\end{gathered}
$$

Écartement $h$ indépendant de l'épaisseur du banc $H$ est le résultat fréquemment observé (fig. 5) pour les fortes épaisseurs de banc (Ladeira et Price, 1981).

Remarque : seules les équivalences ont été mentionnées dans ce qui précède, car cette seule information présente de l'intérêt vis-à-vis de la situation « couloir ». Putot et al. (1999) et Fournigault (2000) explicitent les constantes de proportionnalité entre grandeurs ainsi que la dépendance vis-à-vis du chargement $\lambda$.

\subsubsection{Perspectives pour la caractérisation géologique}

L'approche théorique présentée ci-dessus est validée par le fait qu'elle permet de retrouver des résultats bien connus empiriquement par les géologues, notamment la proportionnalité entre épaisseur de bancs et écartement des fractures, observée pour des épaisseurs de banc inférieures à 1 à $2 \mathrm{~m}$. Elle permet également d'établir une relation entre la densité de fissuration et la nature du matériau, par le biais du paramètre de ténacité. Les méthodes de mesure de cette ténacité, présentées en annexe, ouvrent la voie à une démarche prédictive de l'estimation des variations spatiales de densité de fissuration liée aux variations lithologiques à partir d'essais sur des échantillons prélevés dans les forages. Le succès de cette prévision serait alors bien sûr lié à la représentativité des tests réalisés à l'Actuel, pour estimer les paramètres de fracturation dans les conditions où celles-ci se sont formées.

\section{MODÈLE « COMPOSITE » DE COUCHES COMPÉTENTES ET INCOMPÉTENTES. TRANSITION VERS LE CONCEPT DE COULOIR}

Des interbancs épais de roche incompétente constituant les litages augmentent de manière significative la ductilité de la configuration examinée et donc l'écartement moyen des diaclases. L'influence des paramètres d'épaisseur et de caractéristiques mécaniques du litage a été reconnue par de nombreux spécialistes : Hobbs (1967), Cook et Erdogan (1972), Huang et Angelier (1989), Narr et Suppe (1991), Gross (1993), Wu et Pollard (1995), Ji et Saruwatari (1998).
On a également examiné l'influence du contraste de modules entre banc compétent et litage, pour des confinements variés. Des effets antagonistes semblent s'exercer, faisant apparaître une dominante différente à faible et fort confinement. Des phénomènes similaires semblent avoir été observés dans les structures composites (Martin, 1996).

La présence d'une interface incompétente se traduit dans l'analyse par d'autres manifestations que la simple modification d'un écartement moyen entre diaclases d'un banc compétent donné : le degré de «persistance » d'une diaclase à travers les litages est un élément déterminant. Une forte continuité de la fracture à travers les bancs constitue la diaclase majeure évoquée précédemment.

Des éléments théoriques relativement récents développés pour l'application « matériaux composites » (Leguillon, 1993 ; Martin, 1996 ; Bilteryst, 2000) peuvent aider à situer les conditions de propagation d'une fracture s'approchant normalement d'une interface, susceptible soit de bifurquer parallèlement à celle-ci soit de la traverser complètement (Thiercelin, 1985). Le premier cas pourrait être celui d'une famille de diaclases limitée à un banc compétent, tandis que le second serait celui d'une diaclase majeure, présentant un certain degré de persistance interbancs et susceptible de donner naissance à un couloir. Le modèle de couches sédimentaires indépendantes précédent, associé à une échelle $H$, épaisseur moyenne d'un banc compétent, devient partiellement inadapté.
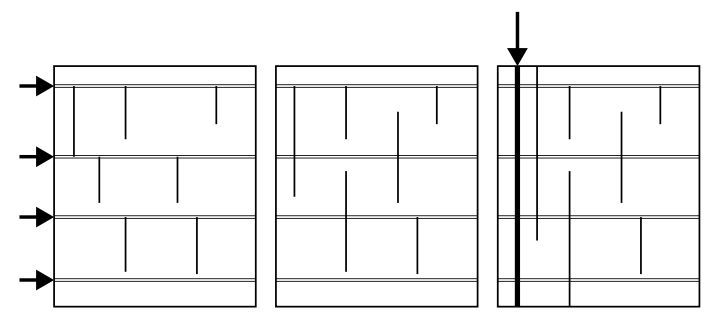

Figure 6

Transition du système de diaclases au couloir de fracturation. À gauche, le système homogène de diaclases : pas de persistance, l'organisation du réseau est perpendiculaire aux interfaces incompétentes (litages) indiquées par les flèches. Ce sont elles qui constituent l'ossature de base.

Au centre, la situation de transition où un certain degré de continuité interbancs est mis en évidence.

À droite, dans le cas du couloir, l'organisation du réseau de fracturation s'effectue parallèlement à la surface libre et la stratification ne joue plus qu'un rôle secondaire.

Transition from a joint set to a fracture swarm.

Left, a homogeneous joint set with no propagation through beds. The joint set is organized normal to the incompetent layers shown by arrows. The stratification controls the orientation of the joint set.

Center, a transition case, where some degree of continuity is assumed.

Right, in the presence of a fractures swarm, the joint set is organized parallel to the free surface and stratification no longer plays a significant role. 
Comme annoncé plus haut, le modèle de couloir décrit ciaprès est, pour ce deuxième cas, davantage pertinent. L'organisation du réseau de fractures s'effectue alors parallèlement à ce nouveau type d'interface constitué par la diaclase majeure dont on peut considérer qu'elle est une surface libre. Nous sommes loin du réseau de diaclases précédent qui s'organisait, quant à lui, perpendiculairement à une interface $a$ priori au contraire très confinée (fig. 6).

\section{COULOIR DE FRACTURATION}

\subsection{Choix de modèles}

Dans le modèle de diaclases, la fissuration était supposée s'effectuer par flexion sous fort confinement avec rôle dissymétrique de la partie en extension et de la partie comprimée.

Pour le modèle de couloir (Chastanet, 2001), la présence d'une surface libre nous incite à imaginer des ruptures successives de type exfoliation, rencontrées dans des situations aussi diverses que l'écaillage des parois d'un puits de forage (Bažant et al., 1993 ; Zheng et al., 1989 ; Ewy et Cook, 1990), les fractures en naissance d'arche parallèlement aux parois d'un tunnel. Le délaminage des composites (Kachanov, 1988) semble également procéder d'un mécanisme voisin. Les théoriciens (Vardoulakis et Mulhaus, 1986 ; Nemat-Nasser et al., 1980) se sont interrogés sur les raisons expliquant la localisation d'une fracture à proximité d'une surface libre plutôt qu'à l'intérieur d'un massif : c'est ce qui distingue le présent phénomène de celui décrit sous le terme de flambement interne (Ashby et Hallam, 1986 ; Kemeny et Cook, 1985). Vardoulakis et Mulhaus (1986) ont également précisé les conditions permettant la sélection d'un mécanisme de type écaillage préférentiellement à un mécanisme de rupture par bande de cisaillement.

Deux types de modèles explicatifs simples (fig. 7) ont été abordés (Chastanet, 2001) :

- La première classe de modèles (Bažant et al., 1993 ; Kachanov, 1988) est celle mettant en œuvre, sous l'effet d'un chargement au loin, le flambage élastique de structures. Celles-ci sont nécessairement élancées et assimilables à des poutres : le principe de minimisation d'énergie invoqué à propos du traitement mécanique du système de diaclases l'est encore ici pour la détermination de l'épaisseur de feuillet la plus probable. Ce raisonnement conduit néanmoins à une estimation d'épaisseur trop faible en regard des faciès observés.

- La seconde classe (Kendall, 1978 ; Evans et al., 1984) envisage les fractures comme résultant de l'action de concentrateurs d'effort, de type plateau (Kendall, 1978) non singuliers, c'est-à-dire n'allant pas jusqu'à exercer un effet très localisé de coin qui serait à l'origine de fissures, quelles que soient les conditions de chargement (Janssen et al., publication en cours). L'effort, canalisé par les concentrateurs, est exercé parallèlement à la surface libre supposée. Il poinçonne une structure élémentaire, susceptible d'être reproduite de façon conceptuelle parallèlement à elle-même pour constituer un massif rocheux semi-infini éventuellement fracturé de manière périodique, la surface libre - diaclase majeure - générant un plan de symétrie. À noter que globalement, sur la largeur de la zone couloir, l'homogénéité et donc la périodicité mentionnée sont en fait mises en défaut en raison d'un éloignement plus ou moins important de la surface libre induisant à coup sûr un gradient d'états. La variable $s$ dite confinement latéral sera justement introduite pour moduler l'état. La périodicité déclarée plus haut n'est donc que locale et approximative.

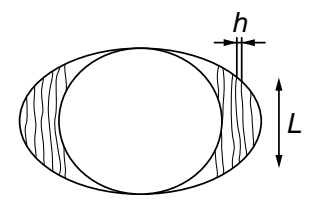

Bažant

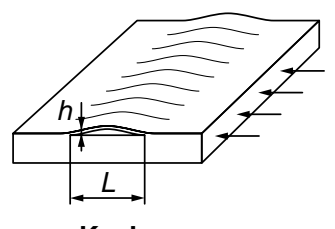

Kachanov
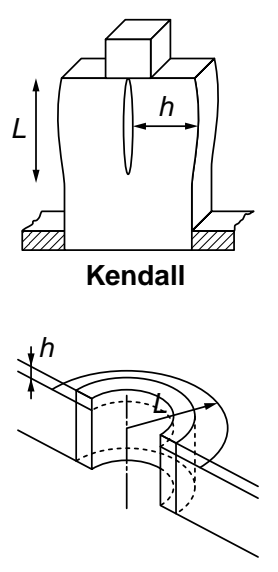

Evans
Figure 7

Modèles étudiés. À gauche, modèles de type flambage élastique ; à droite, modèles de type transition ductile-fragile.

Typical models. Left, examples of models based on elastic buckling; right, examples of models exhibiting a brittleductile transition.

En fait, comme on l'a vu à propos des commentaires relatifs aux modèles appartenant à la première classe (flambage élastique), la seule prise en compte du « chargement au loin » ne permet pas de restituer l'ordre de grandeur des écartements observés. L'effet « guillotine » imposé par le chargement du massif parallèlement et à proximité immédiate de la surface libre fragilise beaucoup la configuration. Au contraire, les concentrateurs de type plateau-poinçon introduisent un confinement propre à inhiber les fissures qui, lorsqu'elles apparaissent, sont plus écartées et généralement plus courtes que dans le cas précédent : la plasticité est alors favorisée. On doit retrouver, comme dans le cas de l'étude des diaclases, un écartement à l'échelle (multiple) d'une dimension de zone plastique $d^{*}$ (Atkins et Mai, 1985). 


\subsection{Description synthétique du modèle en l'absence de confinement latéral}

Du point de vue «technique de calcul », au lieu de considérer, sur la base d'un principe de minimum, l'équidistance probable de fractures sur un modèle d'ensemble constitué de la juxtaposition de motifs périodiques, on envisage ici, sur un motif géométrique isolé, la transition ductile/fragile, c'est-àdire la taille limite $d^{*}$ au-dessous de laquelle seul un mécanisme de type ruine plastique peut se développer, les tailles au-delà du seuil permettant au contraire la croissance d'une fissure. L'écartement le plus probable sera celui réalisant la transition de mécanismes.

De manière plus précise, la transition est envisagée sous deux angles différents : le paramètre échelle (relié à $d$ ) évoqué précédemment et le paramètre « concentration » qui sous-tend en fait une classe de modèles avec extension de plateau $\omega$ plus ou moins grande (fig. 8 ).

Dans la classe précitée, la configuration $\omega / d=0,5$ est celle pour laquelle la fissure apparaît le plus précocement dans la gamme des $d$ croissants. Ce choix de modèle de plateau permet de définir le seuil de fissuration $d^{*}$.
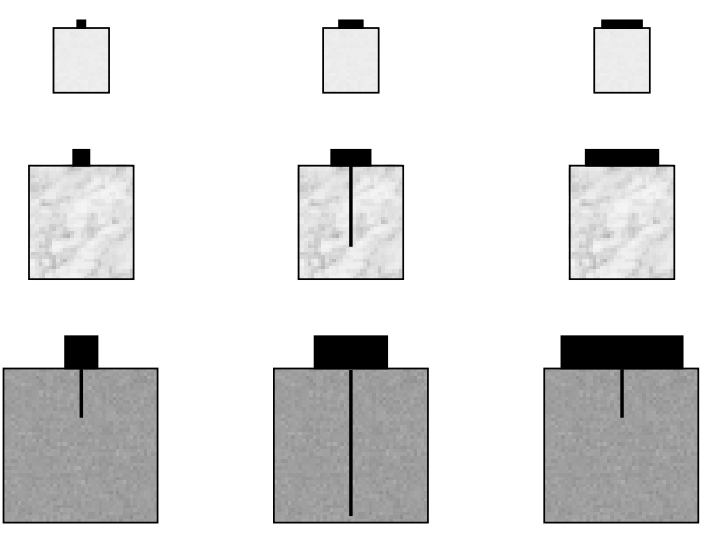

Figure 8

Allure de la fissuration en fonction de la taille $d$ de l'échantillon et de la largeur relative de plateau $\omega / d$.

La taille médiane correspond à un $d$ un peu au-delà du seuil $d^{*}$ pour lequel une fissure de taille indéterminée apparaît avec un plateau $\omega / d=0,5$.

Les échelles $d<d^{*}$ ne peuvent occasionner de fissuration.

Les échelles $d>d^{*}$ mettent en évidence des fissurations d'autant plus développées que le plateau présente un $\omega / d$ proche de 0,5 .

Crack pattern, as a function of sample size, $d$, and relative platen extension, $\omega / d$.

The median size corresponds to a value of $d$ slightly beyond the threshold $d^{*}$ for which a crack of indeterminate length is expected with a relative platen extension $\omega / d=0.5$.

Values of $d<d^{*}$ do not allow cracking.

Values of $d>d^{*}$ exhibit increasingly extensive cracking as the relative platen extension, $\omega / d$, approaches 0.5 .

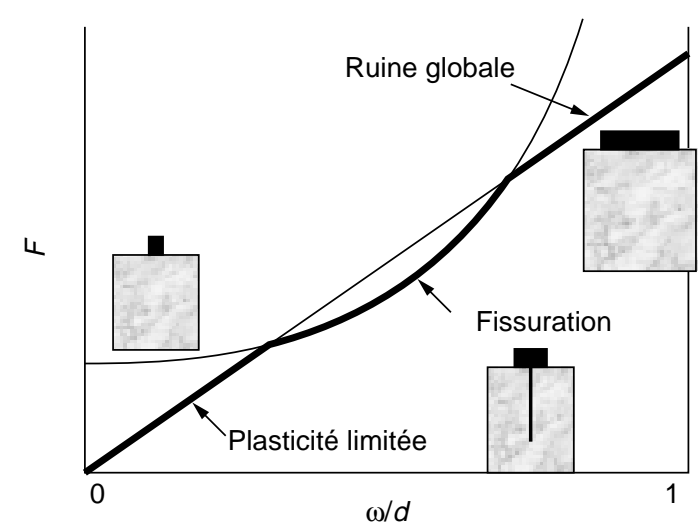

Figure 9

$d$ étant fixé, le mécanisme prévalant, selon la largeur relative du plateau, est celui nécessitant le minimum d'effort $F$ : fissuration privilégiée dans la plage centrale en $\omega / d$.

With d constant, the prevailing mechanism, for a relative platen extension, $\omega / d$, is the one that requires a minimum load, $F$. Cracking will be favored in the central section of $\omega / d$.

Pour $d>d^{*}$, l'intervalle de valeurs $\omega / d$ pour lesquelles la fissuration apparaît dans un modèle de taille $d$ et de plateau $\omega$, s'agrandit avec $d$ en restant centré sur $\omega / d=0,5$ (fig. 9). Des hypothèses seront avancées plus loin pour préciser l'origine physique présumée des concentrateurs. Si l'on autorise une distribution étendue de la taille probable des concentrateurs $(\omega / d$ appartenant à un intervalle d'extension raisonnable centré sur $\omega / d=0,5)$, la transition recherchée est peu supérieure à $d^{*}$, compte tenu de la physionomie de la carte de mécanismes (fig. 9). En revanche, si les concentrateurs sont d'une nature très particulière, soit très locaux (tendance au développement d'une plasticité très confinée), soit très étendus (tendance à une ruine globale), la fissuration n'apparaîtra que plus tardivement. La configuration sera jugée plus ductile.

En l'absence de confinement latéral - ce paramètre de chargement sera envisagé plus loin - la longueur de fissure $L$ est indéterminée, ce qui ne permet pas de préciser dans ce cas le rapport d'aspect $L / h$.

Il est intéressant de noter la valeur analytique explicite de $d^{*}$ (Kendall, 1978) ce qui permet de la situer dans le cadre général défini par Puttick (1979) pour la transition basse (cadre de la mécanique linéaire de la rupture) :

$$
d^{*}=\alpha\left(\frac{K_{I C}}{Y}\right)^{2}
$$

$Y$ est une limite d'écoulement qui est l'analogue de $\sigma_{0} \mathrm{du}$ contexte « diaclases».

- $\alpha$ élevé signifie que la configuration est «naturellement ductile », c'est-à-dire peu propice à la propagation de fractures ; c'est le cas par exemple du poinçonnement d'un massif semi-infini par une sphère. Une valeur typique de l'ordre de 200 est admise pour la transition vers la fissure conique de Hertz. 
- $\alpha$ petit traduit au contraire une certaine aptitude de la configuration au développement d'une fissure ; c'est le cas des éprouvettes entaillées ou des configurations d'usinage pour lesquelles $\alpha$ est voisin de 0,9 .

Dans le cas présent on relève une valeur intermédiaire pour $\alpha$ :

$$
d^{*}=\frac{32}{3}\left(\frac{K_{I C}}{Y}\right)^{2}
$$

On vérifie que celle-ci est proportionnelle à une taille de zone plastique entourant le front de fissure, d'où justification d'une notation $d^{*}$ identique à celle adoptée pour l'étude des diaclases.

Pour ces dernières, le cadre de l'hypothèse 1 :

$$
Y \propto H^{-1 / 2}
$$

nous permet de vérifier que le jeu des paramètres de matériau s'y exprime de manière identique à celui explicité dans le cadre « couloir » (proportionnalité de $h$ et de $d^{*}$ ). La différence réside seulement dans le facteur de proportionnalité $\alpha$ :

- dans le cas des diaclases, le facteur de proportionnalité est $a$ priori élevé et variable puisqu'il consacre des ordres de grandeur de $h$ proches de $H$ plutôt que de $d^{*}$ : en résumé, ce facteur est lié à l'épaisseur du banc et au matériau roche ;

- dans le cas des couloirs, le facteur est modéré et constant puisqu'il est exclusivement lié au matériau !

\subsection{Extension du modèle au confinement latéral $s$}

$s$ mesure la contrainte principale latérale rapportée à une limite conventionnelle d'écoulement $Y$. La prise en compte de ce paramètre se justifie dans la mesure où, s'éloignant de la diaclase majeure, supposée assimilable à une surface libre, on pénètre dans un massif de plus en plus contraint. $s$ pourrait probablement être approché en tenant compte du comportement relaxant dû à la formation des fractures délimitant les blocs constitués de proche en proche, mais cela n'est pas ici notre objectif de déterminer $s(x)$ où $x$ désigne la distance à la surface libre. $s$ étant fixé, correspondant à une certaine distance $x$, la loi $s(x)$ croissante étant supposée connue, on examine l'effet de cette variable sur l'aptitude à la fissuration de la même classe de modèles géométriques envisagée plus haut pour $s=0$.

On a vu (fig. 8 et 9) que, dans le cas $s=0$, l'intervalle en $\omega / d$ pour lequel la fissuration est possible s'étend avec la taille $d$ du modèle. La limite entre mécanismes est notée $\Gamma_{0}$.

Un confinement latéral $s \neq 0$ délimite de même un domaine $\Gamma_{s}$ à droite duquel la fissuration est possible (fig. 10). Dans ce cas, et c'est ce qui le différencie de la situation sans confinement pour laquelle la longueur de fissure était indéterminée, cette longueur de fissure est définie par le rapport d'aspect :

$$
k=\frac{L}{d}
$$

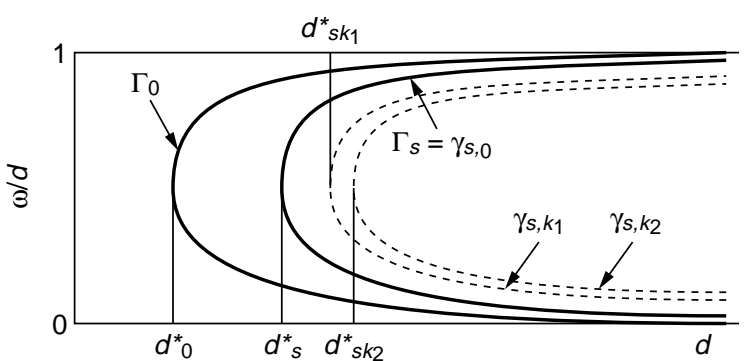

Figure 10

Repères de fissuration en fonction de la largeur de l'échantillon $d$ et de l'extension relative du plateau $\omega / d$.

$\Gamma_{0}$ désigne la limite de fissuration (fissure de longueur indéterminée) pour le confinement nul $(s=0)$.

$\Gamma_{s}=\gamma_{s, 0}$ désigne la limite de fissuration (fissure évanescente) pour le degré de confinement $s$.

$\gamma_{s, k}$ désigne le lieu des classes de modèles de largeur $d$ et d'extension relative de plateau $\omega / d$ pour lesquelles le rapport d'aspect des fissures est $k$.

Cracking boundary limits, as a function of sample width, $d$, and relative platen extension, $\omega / d$.

$\Gamma_{0}$ refers to cracking boundary limit (with an indeterminate crack length) for a confinement parameter $s=0$.

$\Gamma_{s}=\gamma_{s, o}$ refers to cracking boundary limit (crack initiation) for a value s of the confinement parameter.

$\gamma_{s, k}$ refers to the subset of models for which the value of the crack aspect ratio is $k$, the sample width is $d$ and relative platen extension is $\omega / d$.

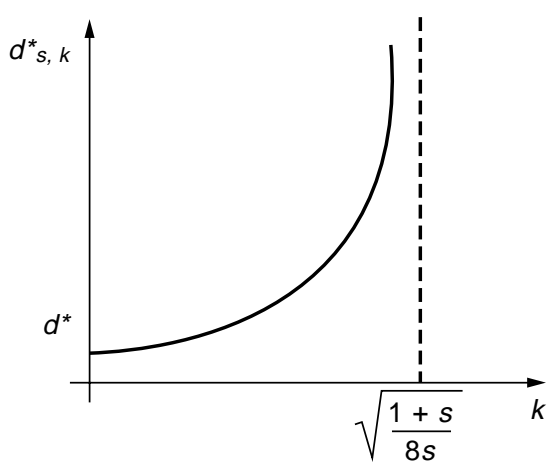

Figure 11

Effet de taille $d_{s, k}^{*}$ de l'échantillon et rapport d'aspect $k$ en fonction du paramètre de confinement $s$.

Sample size effect $d^{*}{ }_{s, k}$ and aspect ratio, $k$, as a function of the confinement parameter, $s$.

Plus précisément $\gamma_{s, k}$ est le lieu des combinaisons $(d, \omega / d)$ pour lesquelles une fissuration de rapport $k$ donné se développe en présence d'un confinement $s$ (fig. 11). En particulier $\gamma_{s, o}=\Gamma_{s}$ contient tous les $\gamma_{s, k}$ définis par l'équation :

$\gamma_{s, k}:\left(\frac{\omega}{d}\right)^{2}-\left(\frac{\omega}{d}\right)+\frac{1}{1+s} \sqrt{\frac{2}{3}\left(\frac{K_{I C}}{Y}\right)^{2} \frac{1}{d}}+2 \frac{s}{1+s} k^{2}=0$ (9) 
Le sommet des courbes $\gamma_{s, k}$ est $d^{*}{ }_{s, k}$ défini par :

$$
d *_{s, k}=\frac{1}{\left(1+s\left(1-8 k^{2}\right)\right)^{2}} \frac{32}{3}\left(\frac{K_{I C}}{Y}\right)^{2}
$$

Pour un confinement $s$ donné, le rapport d'aspect $k$ de la fissure se développant dans un motif de largeur caractéristique $d$ susceptible de prendre n'importe quelle valeur supérieure à $d_{s}^{*}=d^{*}{ }_{s, 0}$ est plafonné, ainsi que l'indique la figure 11, et ceci d'autant plus que $s$ est plus élevé. La ductilité induite par le confinement espace les fissures et limite leur longueur.

\section{Remarques :}

$-s=0$

À $d$ fixé supérieur à $d^{*}$, un choix de valeurs $\omega / d$ proches des limites de plasticité (encourageant la ductilité) :

$$
\frac{\omega}{d} \propto \varepsilon \quad \text { ou bien : } \quad 1-\frac{\omega}{d} \propto \varepsilon
$$

induit des écartements de fractures notablement plus importants que celui obtenu pour $\omega / d=0,5$ mais la longueur de fissure demeure indéterminée. Ceci justifie une certaine variabilité dans les écartements de fractures observées sur le terrain en correspondance avec celle des caractéristiques des concentrateurs supposés.

- $s \neq 0$

Le choix de $\omega / d$ dans le modèle dépend, dès lors qu'on introduit $s$, de la longueur des fractures, paramètre $k: k$ est de ce fait entièrement déterminé par $\omega / d$, à $d$ fixé, ce qui est nouveau par rapport au cas $s=0$. Les variabilités sur $k$ et $\omega$ sont toutes deux alignées sur celle de $\omega / d$.

\section{SYNTHĖSE DES RÉSULTATS}

Moyennant certaines approximations conduites sur la relation exacte, écartement $h$ et rapport de forme $L / h$ ont, pour les couloirs de fracturation, les tendances asymptotiques suivantes, en fonction du confinement $s$ (la valeur $s=10^{-2}$ étant considérée comme une valeur très élevée) :

$$
\begin{aligned}
& s=0 \quad h \propto\left(\frac{K_{I C}}{Y}\right)^{2} \propto d * \\
& s=10^{-2} \quad \frac{L}{h} \propto 1
\end{aligned}
$$

La première configuration $s=0$ (à proximité de la surface libre constituée par la diaclase majeure) correspond précisément au cadre de l'hypothèse 1 lorsque l'on concentre son attention sur la variable écartement $h$ :

$h \infty H \infty d^{*}$ cadre d'hypothèse 1 des diaclases, relation (4a)
La seconde configuration $s=10^{-2}$, assez confinée et susceptible de représenter la marge du couloir, correspond également au cadre de l'hypothèse 1 mais lorsqu'on s'intéresse au rapport d'aspect $L / h$. Le résultat obtenu dans ce cas pour le couloir est à rapprocher de celui obtenu pour les diaclases ainsi qu'indiqué ci-après :

$$
\frac{L}{h} \propto 1 \text { cadre d'hypothèse } 1 \text { des diaclases, relation (4c) }
$$

Finalement, la situation des diaclases, par essence d'allure homogène, réalise un compromis, tantôt sur l'écartement $h$, tantôt sur le rapport d'aspect $L / h$, entre les situations extrêmes attribuées aux couloirs dans une situation de gradient de confinement $s$ :

- en surface libre $(s=0)$

tendance à $h=$ constante

$L / h$ indéterminé ;

- en situation confinée $\left(s=10^{-2}\right)$

tendance à $L / h=$ constante $\quad h$ indéterminé.

De manière plus générale, les confinements intermédiaires relatifs à un état, ni très proche de la diaclase majeure $(s=0)$, ni en marge du couloir $\left(s=10^{-2}\right)$ peuvent approximativement être représentés par les schémas équivalents suivants, où $\alpha$ est un exposant fonction de $s$ qu'il est possible d'identifier numériquement (tableau 1).

TABLEAU 1

Influence du confinement $s$ sur l'écartement $h$ et le rapport de forme $L / h$

Influence of the confinement parameter, $s$, on spacing, $h$, and the aspect ratio, $L / h$

\begin{tabular}{c|c|c}
\hline$s \rightarrow 0 \quad \alpha \rightarrow 2$ & $s \approx 10^{-2} \quad \alpha \approx 0$ & $\begin{array}{c}\text { Configuration ductile } \\
\alpha \text { variable avec } s\end{array}$ \\
\hline$\frac{L}{h} \propto\left(\frac{Y}{K_{I C}}\right)^{2} L$ & $\frac{L}{h} \propto 1$ & $\frac{L}{h} \propto\left(\frac{Y}{K_{I C}}\right)^{\alpha} L^{\alpha / 2}$ \\
\hline$h \propto\left(\frac{K_{I C}}{Y}\right)^{2}$ & $h \propto L$. & $h \propto\left(\frac{K_{I C}}{Y}\right)^{\alpha} L^{1-\alpha / 2}$ \\
\hline
\end{tabular}

\section{Perspectives pour la caractérisation géologique}

Le module présenté ci-dessus permet d'expliquer la formation d'une fracturation localisée en couloirs, à condition de faire intervenir des hétérogénéités préexistantes : une surface libre en subsurface peut être observée le long d'un plan de faille ou le long d'une grande fracture ouverte. De même, le rôle de concentrateur de contraintes peut être tenu par une hétérogénéité locale du matériau, qui engendre une hétérogénéité locale du champ de contraintes. La vérification de ce module à partir de mesures de terrain pourrait être réalisée en analysant de manière détaillée l'évolution spatiale de l'espacement et de la longueur des fractures de part et d'autre du 
« cœur » du couloir fissuré, ainsi que l'identification d'un plan de fracturation préexistant. Dans un cas idéal, il faudrait procéder à une datation relative du plan initial et des fractures du couloir. Ces mesures demandent des affleurements de qualité exceptionnelle.

Si ce modèle se révèle exact, la caractérisation des réservoirs comportant des couloirs fissurés devra reposer en fait essentiellement sur la caractérisation de ces hétérogénéités préexistantes : ce sont elles qui conditionnent la dimension, l'orientation et l'espacement entre les couloirs, et donc la stratégie d'implantation des forages.

\section{CONCLUSIONS}

Le but principal de notre étude était de relier l'écartement typique de fractures à la nature de la roche par l'intermédiaire des propriétés mécaniques, en tenant compte de traits structuraux éventuellement influents (épaisseur de bancs compétents, diaclase majeure). Des situations types ont été imaginées grâce aux observations et interprétations des géologues. Ainsi, on a pu distinguer deux grands types de fractures :

- celles qui sont à l'échelle des bancs ;

- celles qui, présentant un degré de continuité (persistance), s'organisent en un cortège accompagnant une diaclase majeure.

Notre contribution a consisté à caler des modèles de structures fissurées jugés représentatifs, de manière à discerner des tendances sur les écartements moyens. Les résultats sont assez différents selon le type retenu :

- l'épaisseur du banc intervient fortement dans le premier cas, mais les propriétés mécaniques également, sous la forme du groupement habituel $K_{I C} / Y$;

- dans le deuxième cas, la sensibilité au confinement près d'une surface libre est déterminante; le gradient de contraintes conditionne la profondeur des écaillages successifs. Le même paramètre «matériau » évoqué précédemment intervient encore.

\section{RÉFÉRENCES}

Ashby, M.F. et Hallam, S.D. (1986) The Failure of Brittle Solids Containing Small Cracks under Compressive Stress States. Acta Metall., 34, 3, 497-510.

Atkins, A.G. et Mai, Y.W. (1985) Deformation Transitions, in Elastic and plastic fracture, Ellis Horwood Limited (ed.), Chichester, England.

Atkinson, B.K. (1984) Subcritical Crack Growth in Geological Materials. J. Geophys. Res., 89, 4077-4114.

Atkinson, B.K. (1987) in Fracture Mechanics of Rocks, Academic Press Geology Series, Harcourt Brace Jovanovitch.

Auzias, V. (1995) Contribution à la caractérisation tectonique des réservoirs fracturés, Thèse de troisième cycle, univ. Montpellier II.

Bahat, D. (1991) Burial Jointing, Syntectonic Jointing, Uplift Jointing, in Tectonofractography, Springer-Verlag (ed.), Berlin, Heidelberg, New York.
Bai, T. et Gross, M.R. (1999) Theoretical Analysis of Cross-Joint Geometries and their Classification. Journal of Geophysical Research, 104, B1, 1163-1177.

Bažant, Z.P., Lin, F.B. et Lippmann, H. (1993) Fracture Energy Release and Size Effect in Borehole Breakout. Int. J. Num. \& An. Methods in Geomech., 17, 1-14.

Bearman, R.A. (1999) The Use of the Point Load Test for the Rapid Estimation of Mode I Fracture Toughness. Int. J. Rock Mech. Min. Sci. \& Geomech. Abstr., 36, 257-263.

Bilteryst, F. (2000) Une approche énergétique des phénomènes de décohésion et de multifissuration dans les composites. Thèse de doctorat, université Paris VI.

Chastanet, J. (2001) Fracturation naturelle d'un massif rocheux : couloirs de fracturation, Rapport interne IFP 55739.

Cook, T.S. et Erdogan, F. (1972) Stresses in Bonded Materials with a Crack Perpendicular to the Interface. Int. J. Eng. Sci., 10, 677-697.

Evans, A.G., Palmer, A.C., Goodman, D.J., Ashby, M.F., Hutchinson, J.W., Ponter, A.R.S., Williams, G.J. (1984) Indentation Spalling of Edge-Loaded Ice Sheets. IAHR Ice Symposium Hamburg.

Ewy, R.T. et Cook, N.W. (1990) Deformation and Fracture around Cylindrical Openings in Rock-II. Initiation, Growth and Interaction of Fractures. Int. J. Rock Mech. Min. Sci. \& Geomech. Abstr., 27, 5, 409-427.

Fischer, M.P., Gross, R.M., Engelder, T. et Greenfield, R.J. (1995) Finite-Element Analysis of Stress Distribution around a Pressurized Crack in a Layered Elastic Medium: Implication for the Spacing of Fluid-Driven Joints in Bedded Sedimentary Rock. Tectonophysics, 247, 49-64.

Fournigault, D. (2000) Détermination d'une relation définissant un écartement moyen de diaclases, Rapport interne IFP 53199.

Francfort, G.A. et Marigo, J.J. (1993) Stable Damage Evolution in a Brittle Continuous Medium. Eur. J. of Mech., A /Solids, 12, 2, 149-189.

Francfort, G.A. et Marigo, J.J. (1998) Revisiting Brittle Fracture as an Energy Minimization Problem. J. Mech. Phys. Solids, 46, 8, 1319-1342.

Gross, M.R. (1993) The Origin of Cross Joints: Examples from the Monterey Formation, Santa Barbara Coastline, California, Journal of Structural Geology, 15, 6, 737-751.

Hobbs, C.W. (1967) The Formation of Tension Joints in Sedimentary Rocks: an Explanation. Geological Magazine, 104, 550-556.

Huang, Q. et Angelier, J. (1989) Fracture Spacing and its Relation to Bed Thickness. Geological Magazine 126, 355-362.

Janssen, C., Wagner, F.C., Zang, A. et Dresen, G., Fracture Process Zone in Granite. A Microstructural Analysis. En cours de publication au Geolog. Rundsch., Potsdam, Allemagne.

Ji, S. et Saruwatari, K. (1998) A Revised Model for the Relationship between Joint Spacing and Layer Thickness. Journal of Structural Geology, 20, 11, 1495-1508.

Kachanov, L.M. (1988) in Delamination Buckling of Composite Materials, Kluwer Academic Publishers, Dordrecht, Netherlands.

Kemeny, J. et Cook, N.G.W. (1985) Formation and Stability of Steeply Dipping Joint Sets. 26th US Symposium on Rock Mechanics/Rapid City.

Kendall, K. (1978) Complexities of Compression Failure. Proc. R. Soc. Lond. A., 361, 245-263.

Lachenbruch, A.H. (1961) Depth and Spacing of Tension Cracks. Journal of Geophysical Research, 66, 12, 4273-4292.

Ladeira, F.L. et Price, N.J. (1981) Relationship between Fracture Spacing and Bed Thickness. Journal of Structural Geology, 3, 2, 179-183. 
Leguillon, D. (1993) Asymptotic and Numerical Analysis of a Crack Branching in Non-Isotropic Materials., Eur. J. Mech. A/ Solids, 12, 1, 33-51.

Martin, E. (1996) Interaction fissure/interface dans les matériaux fragiles. Application à l'étude du comportement à la rupture de matériaux composites à matrice non organique. Habilitation à Diriger des Recherches, université de Bordeaux I.

Narr, W. et Suppe, J. (1991) Joint Spacing in Sedimentary Rocks. Journal of Structural Geology, 13, 9, 1037-1048.

Nemat-Nasser, S., Sumi, Y., Keer, L.M., (1980) Unstable Growth of Tension Cracks in Brittle Solids: Stable and Unstable Bifurcations, Snap through, and Imperfection Sensitivity. International Journal of Solid Structures, 16, 1017-1035.

Pascal, C., Angelier, J., Cacas, M.C. et Hancock, P.L., (1997) Distribution of Joints: Probabilistic Modelling and Case Study near Cardiff, Wales, UK. Journal of Structural Geology, 19, 10, 1273-1284

Pollard, D. et Aydin, A., (1988) Progress in Understanding Jointing over the Past Century. Geological Society of America Bulletin, 100, 1181-1204.

Putot, C., Fournigault, D. et Cacas, M.C. (1999) Relationship between Joint Spacing and Layer Thickness. A Model Based on Linear Elastic Fracture Mechanics. Second Euroconference on Rock Physics and Rock Mechanics.
Puttick, K.E (1979) Size Effects in Brittle Fracture. ICM 3, Cambridge UK.

Rives, T. (1992) Mécanismes de formation des diaclases dans les roches sédimentaires : Approche expérimentale et comparaison avec quelques exemples naturels. Thèse de troisième cycle, univ. Montpellier II.

Scholz, C.H. (1990) in The Mechanics of Earthquakes and Faulting, Cambridge University Press.

Thiercelin, M. (1985) Instabilité de fracture en mode I. Colloque CNRS Grenoble, 69-88.

Vardoulakis, I. et Mulhaus, H.B. (1986) Technical Note: Local Rock Surface Instabilities. Int. J. Mech. Min. Sci. \& Geomech. Abstr., 23, 5, 379-383.

Whittaker, B.N., Singh, RN, Sun, G. (1982) Rock Fracture Mechanics. Principles, Design and Applications. Elsevier, London.

Wu, H. et Pollard, D.D. (1995) An Experimental Study of the Relationship between Joint Spacing and Layer Thickness. Journal of Structural Geology, 17, 887-905.

Zheng, Z., Kemeny, J. et Cook, N.G.W. (1989) Analysis of Borehole Breakouts. J. Geoph. Res., 94, B6, 7171-7182.

Manuscrit final reçu en août 2001 


\section{ANNEXE 1}

\section{ÉLÉMENTS DE SYNTHĖSE ET IMAGE FINALE DU COULOIR DE FRACTURATION}

Modèles élastiques et modèles de transition (la dépendance exponentielle en $\alpha$ n'est qu'approximative)

\begin{tabular}{c|c}
$\begin{array}{c}\text { Chargement au loin } \\
\text { Gradients élevés, effet de « guillotine» } \\
\text { Fractures longues et rapprochées (configuration fragile) } \\
\text { Modèle de Kachanov }\end{array}$ & $\begin{array}{c}\text { Contrainte locale } \\
\text { Concentrateurs, type poinçon-plateau }\end{array}$ \\
\hline$\frac{L}{h} \propto\left(\frac{E}{K_{I C}}\right)^{2 / 5} L^{1 / 5}$ & $\begin{array}{c}\text { Fractures distantes et courtes (configuration plastique) } \\
\text { Extension modèle de Kendall }\end{array}$ \\
$h \propto\left(\frac{K_{I C}}{E}\right)^{2 / 5} L^{4 / 5}$ & $\frac{L}{h} \propto\left(\frac{Y}{K_{I C}}\right)^{\alpha} L^{\alpha / 2}$ \\
\hline
\end{tabular}

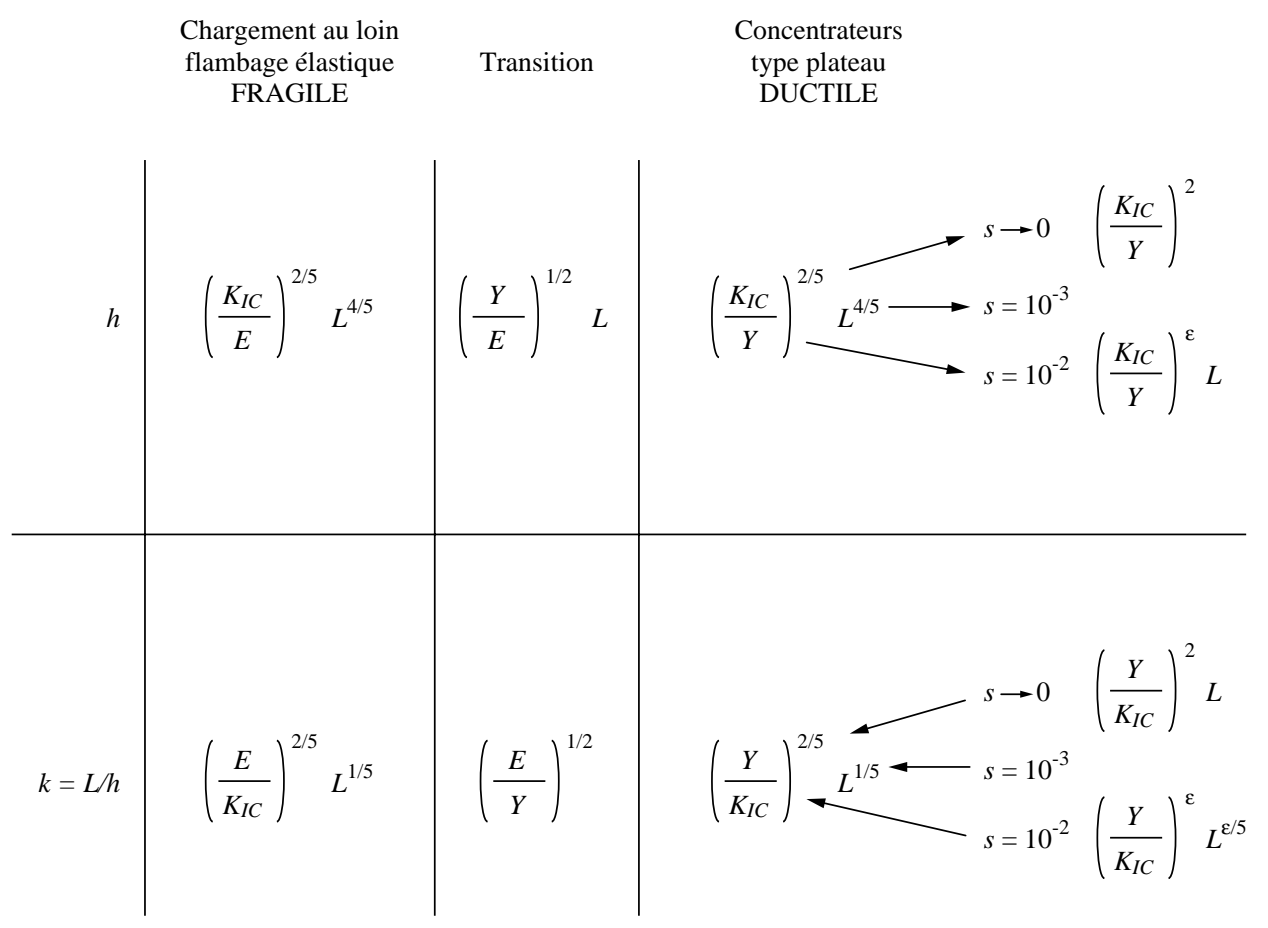

Figure A1

Rôles respectifs joués par le module élastique $E$ et la limite d'écoulement conventionnelle $Y$ dans les modèles. Respective influence of the elastic modulus, $E$, and the conventional yield limit, $Y$, in the different models. 
Valeurs de $L / h$ et facteurs d'échelle selon $L$, en fonction des différents modèles

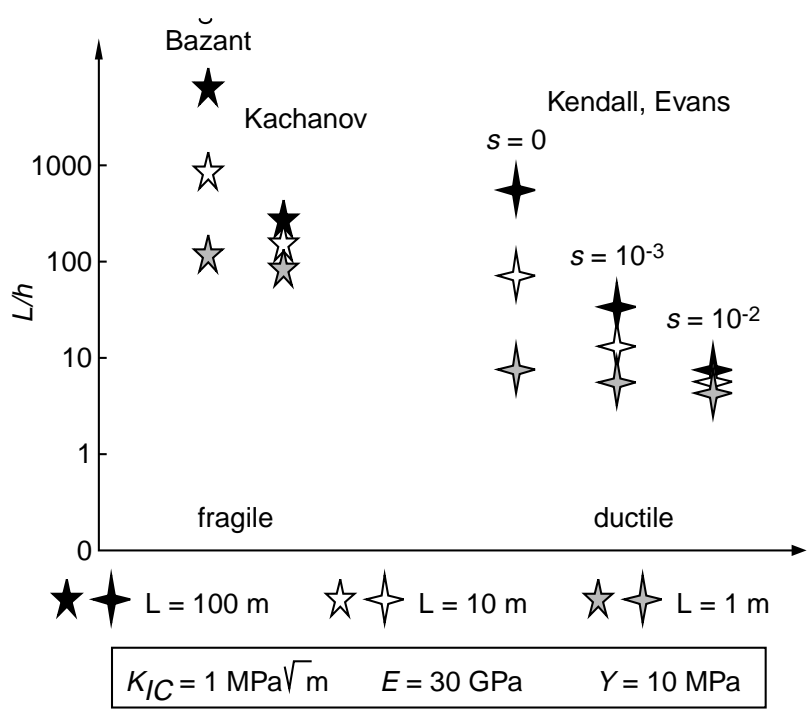

Valeurs de $h$ et facteurs d'échelle selon $L$, en fonction des différents modèles

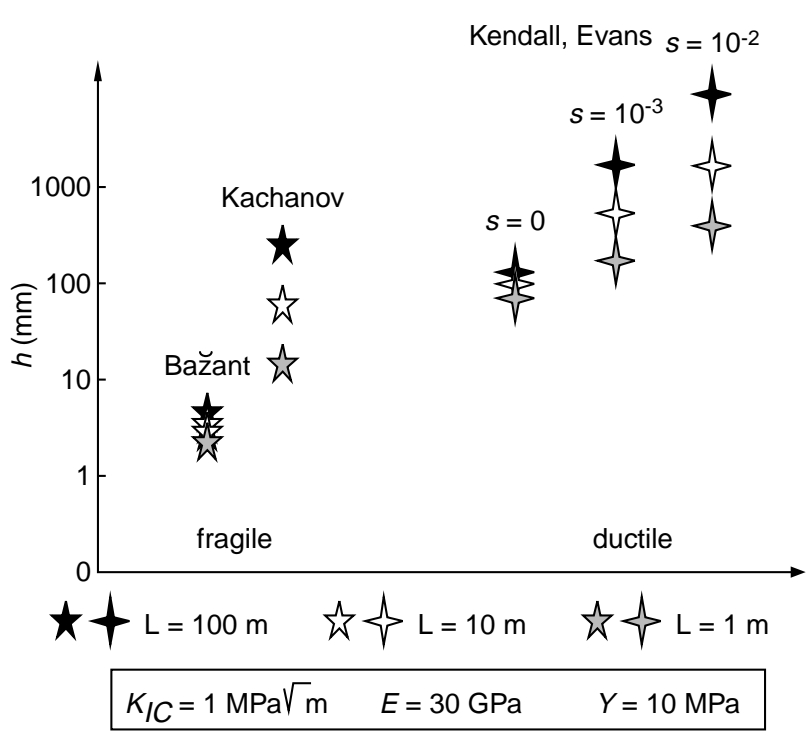

Figure A2

Typologie des fractures, selon les modèles.

Fracture typology, according to the models.

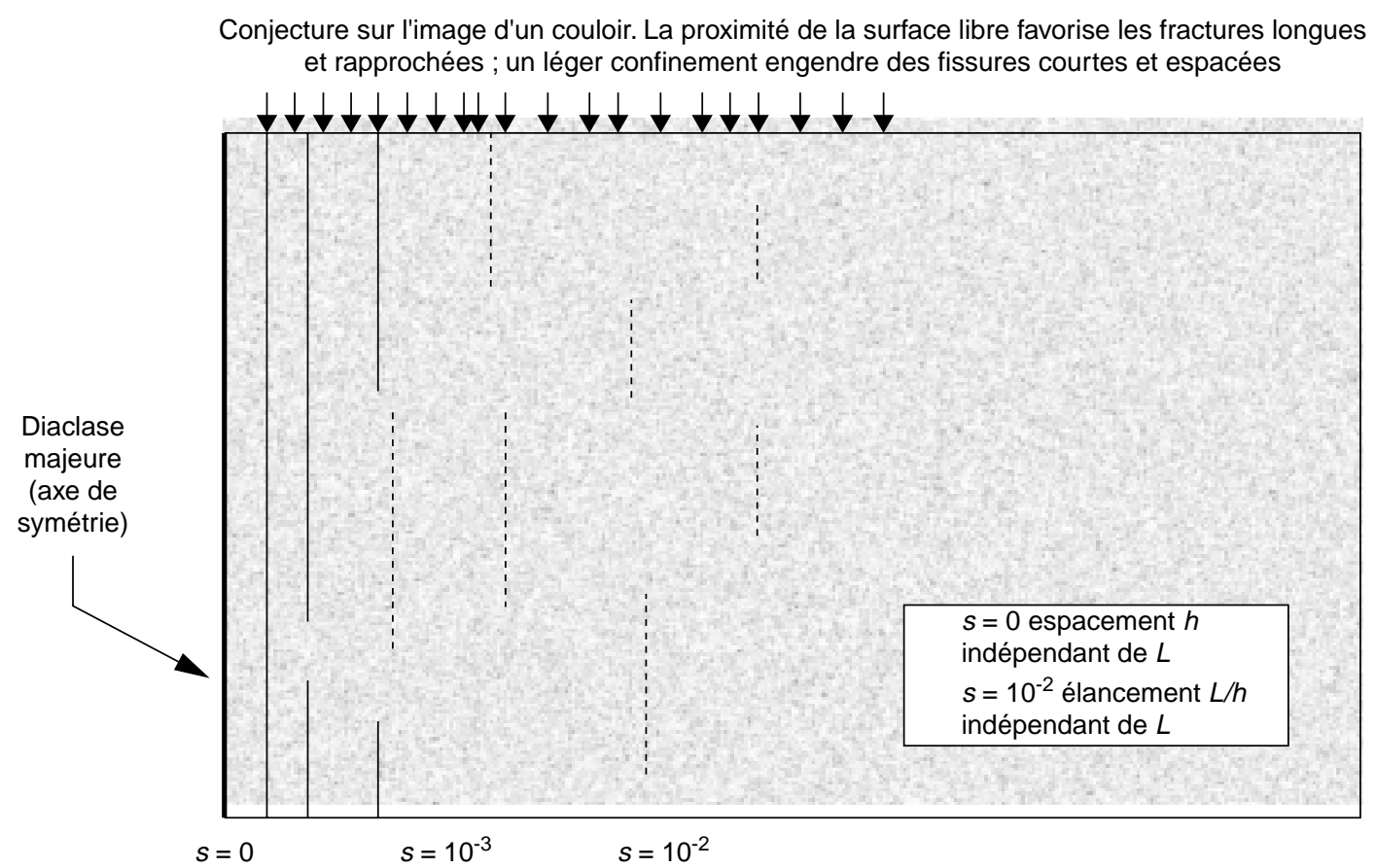

Figure A3

Image finale du couloir.

Final state of the fracture swarm. 


\section{ANNEXE 2 \\ CROISSANCE SOUS-CRITIQUE DE FISSURES DANS LES ROCHES}

On pourra, par exemple, se reporter à Atkinson (1984) pour un état de l'art concernant la description des mécanismes microstructuraux conditionnant la propagation des fractures dans les roches. Nous nous bornerons, ici, à quelques résultats généraux extraits de la publication précitée, concernant les ordres de grandeur de ténacité (fig. A4).

En général, pour les roches siliceuses, plus la proportion de quartz sera faible et plus la teneur en minéraux ferromagnésiens sera importante, plus la roche sera tenace. Des influences complexes liées à la porosité, la taille de grain, etc., viennent brouiller ce schéma de principe.

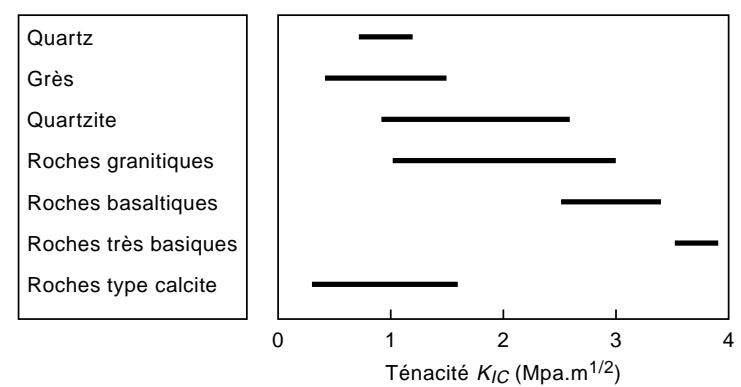

Figure A4

Variation de la ténacité en mode I avec le type de roche. Variation of Mode I toughness with rock type.

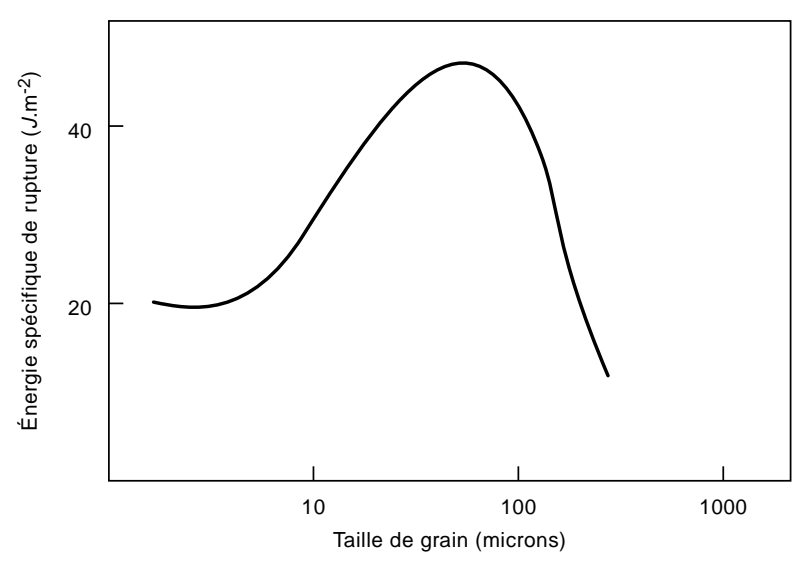

Figure A5

Diagramme schématique montrant l'influence de la taille de grain sur l'énergie spécifique de fracturation.

Simplified diagram showing the influence of grain size on specific work of fracture propagation.

\section{Influence de la taille de grain}

De manière générale, on observe une augmentation de ténacité avec la taille de grain jusqu'à une taille critique au-delà de laquelle une inversion de tendance se produit (fig. A5).

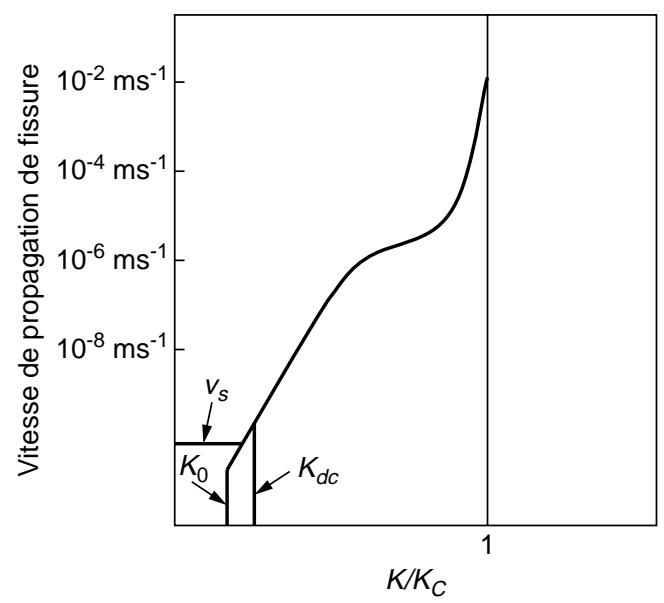

Figure A6

Diagramme schématique montrant l'influence du facteur d'intensité de contraintes critique sur la croissance de fissure dans le domaine sous-critique, pour une roche en conditions de corrosion sous contrainte (d'après Atkinson, 1984).

$K_{I C}$ est la ténacité ou facteur d'intensité de contrainte critique. $K_{0}$ est la limite de croissance en comportement de corrosion sous contrainte.

$v_{s}$ est le seuil de vitesse associé au processus de dissolution au front de fissuration.

$K_{d c}$ est la limite au-dessous de laquelle le fluage par diffusion peut contribuer à la vitesse de déformation et émousser le front macroscopique.

Le comportement de type 1 est lié à l'activité de corrosion au front de fissuration.

Le comportement de type 2 est régi par la possibilité de transport de produits réactifs au front.

Le comportement de type 3 est sous le contrôle d'une rupture thermoactivée pratiquement indépendante de l'environnement chimique.

Schematic diagram showing the influence of the stress intensity factor on subcritical crack growth behavior in a rock undergoing stress corrosion (after Atkinson, 1984).

$K_{I C}$ is the toughness or critical stress intensity factor.

$K_{0}$ is the stress corrosion crack growth limit.

$v_{s}$ is the crack velocity threshold due purely to dissolution at the crack tip.

$K_{d c}$ is the crack growth limit below which bulk diffusion creep dominates deformation behavior.

Region 1 behavior is controlled by the rate of stress corrosion reactions at crack tips.

Region 2 behavior is controlled by the transport rate for reactive species to crack tips.

Region 3 behavior is controlled by thermally activated bond rupture which is largely independent of chemical environment. 
L'accroissement de la ténacité avec la taille de grain résulte de la transition d'un régime de rupture intergranulaire typique des matériaux à grain fin vers un mécanisme de rupture transgranulaire spécifique des matériaux à grain plus grossier. Le plafonnement a été expliqué par l'activité des microfissures accompagnant la macrofissure. Celles-ci sont activées dès lors que les concentrations de contrainte locales peuvent s'exercer de manière significative grâce à l'augmentation de taille de grain. La formation de ces microfissures absorbe de l'énergie, ce qui justifie l'augmentation de ténacité. Lorsque la microfissuration est suffisamment développée, peu d'énergie supplémentaire est nécessaire pour accroître la longueur de la macrofissure, ce qui explique la baisse finale de ténacité.

La fissuration sous-critique, et tout particulièrement à de faibles vitesses de propagation, est associée aux mécanismes intergranulaires tandis que les vitesses élevées, proches de la valeur critique, font partie du domaine de la fissuration transgranulaire.

Certains ont émis l'hypothèse que l'accroissement de ténacité avec la taille de grain pourrait correspondre à la création de fissures branchées progressant à faible vitesse.

De nombreux facteurs entrent en conflit et compliquent l'analyse mais en règle générale, l'énergie de rupture aux joints de grain est inférieure à l'énergie de clivage des grains, en raison des impuretés, porosités et du manque de cohésion entre les contours de grain. De plus, l'énergie spécifique de rupture pour les grandes tailles de grain est inférieure à celle correspondant aux petites tailles de grain, cela dû à une concentration de défauts plus élevée dans le cas des grandes tailles.

\section{Limite de croissance sous-critique}

Le facteur « vitesse de propagation » a été peu évoqué dans cette communication, dont les résultats font référence au facteur d'intensité de contraintes critique $K_{I C}$, mais il est important pour le géophysicien de se rendre compte de l'importance de cette donnée. Celui-ci s'intéressera tout particulièrement aux très faibles vitesses. Il s'interrogera en particulier sur l'existence d'un seuil $K_{0}$ de non-fissuration qui, lorsqu'il existe, est de l'ordre de 10 à $20 \%$ de $K_{I C}$. En fait, comme l'indique la figure $\mathrm{A} 6$, les différents mécanismes susceptibles d'entraîner la progression de la fissure ont chacun un seuil d' « inactivité » au-dessous duquel leur participation ne peut être envisagée. Certaines expérimentations permettant de reconnaître les seuils ont été conduites pour des vitesses aussi faibles que $10^{-12} \mathrm{~ms}^{-1}$.

Le processus de cicatrisation (en l'absence de contrainte) fait l'objet d'études expérimentales spécifiques, fort délicates, puisqu'elles concernent des vitesses aussi faibles que $10^{-15}$ à $10^{-18} \mathrm{~ms}^{-1}$. 


\section{ANNEXE 3}

\section{LIEN ENTRE TÉNACITÉ DES ROCHES ET CARACTÉRISTIQUES À LA RUPTURE}

À titre d'information générale, il est utile de constater que les caractéristiques à la rupture en traction obtenues avec l'essai brésilien sont sensiblement proportionnelles aux valeurs de ténacité mesurées selon la méthode rapide décrite par Bearman (1999). Le tableau A1, inspiré de Bearman (1999), illustre cette comparaison.

\section{TABLEAU A1}

Propriétés mécaniques de roches expérimentées par Bearman (1999)

Mechanical properties of the rock types tested by Bearman (1999)

\begin{tabular}{|c|c|c|c|c|c|}
\hline Type de roche & $\begin{array}{l}\text { Ténacité } \\
\left(\mathrm{MPa} \cdot \mathrm{m}^{1 / 2}\right)\end{array}$ & $\begin{array}{c}\text { Résistance } \\
\text { rupture traction } \\
(\mathrm{MPa})\end{array}$ & $\begin{array}{c}\text { Résistance } \\
\text { compression uniaxiale } \\
\text { (MPa) }\end{array}$ & $\begin{array}{l}\text { Module d'Young } \\
\text { (GPa) }\end{array}$ & $\begin{array}{l}\text { Coefficient } \\
\text { de Poisson }\end{array}$ \\
\hline Calcaire Middleton & 0,73 & 3,8 & 48 & 27,5 & 0,23 \\
\hline Calcaire Harrycroft & 0,82 & 4,6 & 53 & 25,4 & 0,25 \\
\hline Grès Montcliffe & 1,18 & 6,1 & 76 & 15,9 & 0,13 \\
\hline Calcaire Wredon & 1,69 & 10,1 & 157 & 57,2 & 0,28 \\
\hline Granite Penryn & 1,83 & 10,6 & 132 & 39,1 & 0,29 \\
\hline Grès Pennant & 2,10 & 14,0 & 162 & 39,1 & 0,31 \\
\hline Andésite Whitwick & 2,17 & 14,5 & 139 & 63,6 & 0,23 \\
\hline Diorite Bolton Hill & 2,21 & 15,8 & 129 & 54,4 & 0,34 \\
\hline Greywacke Ingleton & 2,38 & 15,2 & 226 & 57,0 & 0,17 \\
\hline Quartzite Nuneaton & 2,44 & 13,0 & 139 & 36,4 & 0,24 \\
\hline Diorite Cliffe Hill & 2,77 & 18,4 & 275 & 64,2 & 0,28 \\
\hline Greywacke Cornish & 3,15 & 15,4 & 165 & 49,6 & 0,25 \\
\hline
\end{tabular}




\section{ANNEXE 4 \\ MÉTHODES DE MESURE DE LA TÉNACITÉ}

Une revue des différentes méthodes comportant spécificités et limitations a été effectuée par Whittaker et al. (1982). Les méthodes permettant de mesurer la ténacité des roches en mode I sont nombreuses. Cette diversité a commencé à se manifester à la fin des années 70, époque à laquelle la détermination de la ténacité commençait à correspondre à un réel besoin.

De nos jours, l'ISRM (International Society for Rock Mechanics) préconise deux types d'essai. En dépit de la standardisation de la mesure de ténacité, l'usage de ce genre d'essai pour caractériser la roche n'est pas très répandu. Les raisons essentielles à cela sont le temps de préparation de l'échantillon, les ruptures prématurées dues à des défauts ainsi que la difficulté de réalisation d'entailles dans la limite des tolérances prescrites.

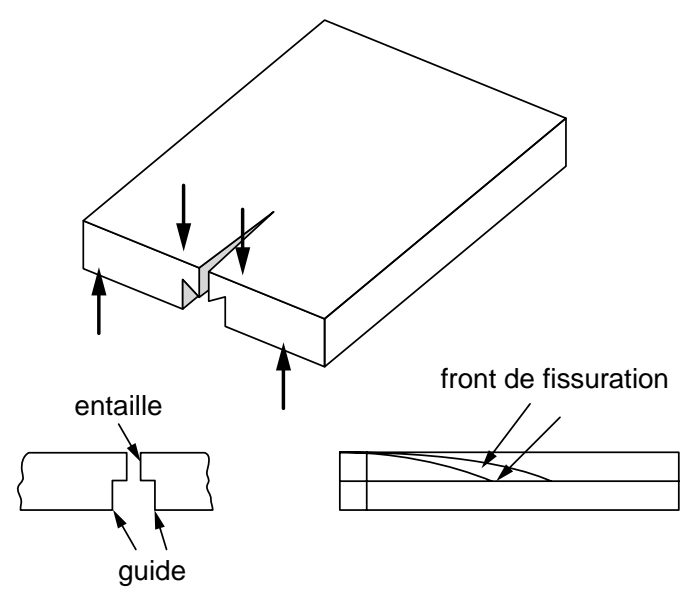

Figure A7

Dispositif expérimental permettant une mesure de ténacité par la méthode de la « double torsion».

Schematic drawing of a double torsion measurement to determine the toughness.

\section{Exemple de dispositif de mesure "fine "}

Atkinson (1984) présente en détail un dispositif expérimental dû à Evans, dit méthode de la double torsion (fig. A7) constitué d'un échantillon en forme de plaque épaisse préentaillée sur un bord et sollicitée en flexion quatre points au voisinage du bord. Le contrôle de la vitesse de fissuration - il s'agit bien d'une mesure des caractéristiques de propagation souscritique - s'effectue en imposant un historique de relaxation de la flexion. Comme pour beaucoup d'expérimentations, le problème principal de ce type d'essai est le manque de raideur du dispositif de chargement. Un deuxième paramètre déterminant est la géométrie de la rainure constituant le guide de la fissuration. Les deux cas limites sont les suivants :

- si le guide est très étroit, la macrofissure est astreinte à suivre un plan assez bien défini ;

- à l'autre extrême, un guide large laisse à la fissure toute latitude pour choisir le cheminement nécessitant le moins d'énergie possible ; dans ce dernier cas le caractère intragranulaire ou transgranulaire de la fissuration est le fait de la roche et non du guidage.

Une expression analytique permet de lier facteur d'intensité des contraintes et vitesse en cours d'essai.

\section{Exemple de mesure rapide sur le terrain}

Bearman (1999) présente une méthode destinée à l'utilisation dans le domaine minier pour apprécier l'aptitude à la fragmentation des roches. Le paramètre ténacité se révèle essentiel. Cette mesure rappelle notamment l'usage bien connu de l'essai brésilien sur disque chargé en compression et dont la rupture s'effectue en traction.

L'essai proposé est adapté au prélèvement de carottes de minerai. Il est également recommandé par l'ISRM. La méthode utilise comme intermédiaire la contrainte de traction résultant de l'application d'une force de poinçonnement, soit sur le diamètre d'un échantillon cylindrique, soit dans l'axe du cylindre. L'essai est également possible sur un échantillon de forme vaguement parallélépipédique. La ténacité est déduite de l'effort à rupture et d'une dimension caractéristique de l'échantillon. 